\title{
Response timing in the lunge and target change in elite versus medium-level fencers
}

\author{
MARCOS GUTIERREZ-DAVILA ${ }^{1}$, FRANCISCO J. ROJAS ${ }^{1}$, RAQUEL ANTONIO ${ }^{2}$, \& \\ ENRIQUE NAVARRO ${ }^{2}$
}

\author{
${ }^{1}$ Faculty of Sports Sciences, University of Granada, Granada, Spain, and ${ }^{2}$ Faculty of Physical Activity and Sport Sciences, \\ Technical University of Madrid, Madrid, Spain
}

\begin{abstract}
The aim of the present work is to examine the differences between two groups of fencers with different levels of competition, elite and medium level. The timing parameters of the response reaction have been compared together with the kinetic variables which determine the sequence of segmented participation used during the lunge with a change in target during movement. A total of 30 male sword fencers participated, 13 elite and 17 medium level. Two force platforms recorded the horizontal component of the force and the start of the movement. One system filmed the movement in $3 \mathrm{D}$, recording the spatial positions of 11 markers, while another system projected a mobile target over a screen. For synchronisation, an electronic signal enabled all the systems to be started simultaneously. Among the timing parameters of the reaction response, the choice reaction time (CRT) to the target change during the lunge was measured. The results revealed differences between the groups regarding the flight time, horizontal velocity at the end of the acceleration phase, and the length of the lunge, these being higher for the elite group, as well as other variables related to the temporal sequence of movement. No significant differences have been found in the simple reaction time or in CRT. According to the literature, the CRT appears to improve with sports practice, although this factor did not differentiate the elite from medium-level fencers. The coordination of fencing movements, that is, the right technique, constitutes a factor that differentiates elite fencers from medium-level ones.
\end{abstract}

Keywords: Biomechanics, motor control, fencing, reaction response, target change

\section{Introduction}

In fencing, the lunge constitutes the most common technical movement of attack. For the lunge, after preparatory movements, the fencer advances from a position called en garde, where the two feet are in contact with the floor and the sword arm is slightly flexed (Figure 1a). Under a stimulus, usually the action of the opponent, the fencer begins to exert force with the rear foot, accelerating forwards with a timing sequence usually started with a thrust of the weapon arm, and a forward step with the front foot (Stewart \& Kopetka, 2005; Figure 1b,c). This acceleration phase ends when the rear foot stops to push against the floor (Figure 1d). Next, a flight phase normally follows, ending with the contact of the front foot on the floor (Figure 1e). Finally, after reaching the target, the force exerted by the front foot brakes the horizontal velocity of the fencer (Figure 1f).

During the time that the acceleration lasts, the fencer in these particular situations makes certain adjustments to change the aim according to the unpredictable response of the opponent. In this situation, the fencer needs to select a response from various options (i.e. choice reaction time [CRT]) to process the information, to slow the initial motor action and to change target while accelerating forward. This is a CRT that necessarily determines the timing parameters of the reaction response time (RRT) and the horizontal forces exerted on the floor. As a consequence, the difficulty of coordinating the technical actions of the legs and the weapon arm during the lunge increases, this

Correspondence: Francisco Javier Rojas, Faculty of Sport Sciences, University of Granada, c/Alfacar s/n, 18071 Granada, Spain. E-mail: fjrojas@ugr.es 


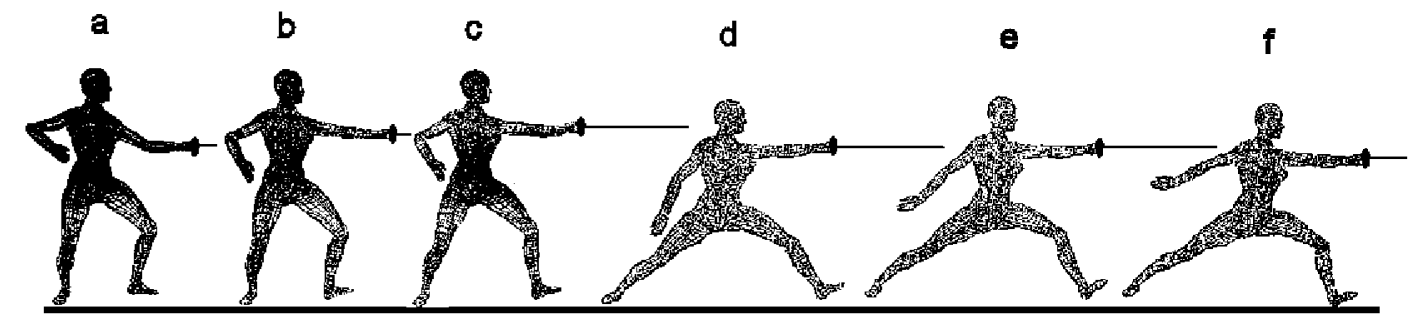

Figure 1. Images of the sequence of a long lunge in fencing.

being especially relevant in fencing performance (Williams \& Walmsley, 2000b; Yiou \& Do, 2000).

The importance that CRT has in fencing has been demonstrated in different studies, some comparing the timing parameters of the RRT between elite and novice fencers (Borysiuk \& Waskiewicz, 2008; Williams \& Walmsley, 2000a) and others comparing fencers with athletes of other sports (Dogan, 2009; Guizani et al., 2006). In all these cases, the timing components of the RRT have been recorded in situations where the fencer responds to different stimuli starting from a position of en garde (standstill). On the contrary, few works have analysed the CRT in situations where the fencer is in movement and has to change targets. In this sense, Williams and Walmsley (2000a), using a protocol of target change, have shown that elite fencers are faster than novices in RT and that the execution of the target change poses no difficulty. Di Russo, Taddei, Aprile, and Spinelli (2006), comparing expert fencers versus control, reported that fencers had a greater capacity to change target to another more appropriate one, being quicker to discriminate the correct stimulus.

Most studies comparing elite and novice fencers report that the CRT is significantly shorter for elite fencers (Borysiuk \& Waskiewicz, 2008; Williams \& Walmsley, 2000a). The finding has been used to indicate the relevance that CRT has in fencing performance, and has even been considered one of the main indicators of talent among fencers (Borysiuk \& Cynarski, 2010). Despite the agreement concerning the importance of CRT in fencing, the notable difference among groups sampled in terms of the quantity of practice due to experience could constitute the causal factor of the differences found. The fact that the reaction time (RT) reduces with practice confirms this hypothesis (Sosnoff \& Samson, 2007; Utley \& Astill, 2008). With the aim of reducing the effect of sports experience, in the present work, the timing parameters of the RRT were compared between two groups of fencers of different competition levels (elite and medium level) but with similar experience. Among the timing parameters of the RRT, the CRT was recorded for target changes during a lunge. Certain factors that determine movement coordination were recorded to compare the technical execution between groups. The complexity of the action, due to the change in the target, leads us to propose that the differences between groups lie in the technical factors related to movement coordination.

\section{Methods \\ Participants}

A total of 30 male sword fencers participated in this study. Of these, 13 were considered elite, forming part of the National Spanish Sword-Fighting Team, and have participated at least in four international competitions of absolute category). The other 17 participants were considered medium level, since they were regular participants during the last four years of the National Spanish Ranking and did not go beyond the 62 qualifying rounds. Table I lists the values of central trend and level of significance for the features of the two groups regarding height, weight, age and years of practice (experience). All participants gave informed consent to participate in the study following the rules of the Ethics Commission of the University of Granada (Spain).

\section{Materials and apparatus}

Two Dinascan/IBV force platforms (Instituto de Biomecánica de Valencia, Valencia, Spain) of $0.6 \times 0.37 \mathrm{~m}$, at $500 \mathrm{~Hz}$, were placed under the feet

Table I. Descriptive and interferential statistics of weight, height, age and years of experience for the elite and medium-level group

\begin{tabular}{|c|c|c|c|}
\hline Variables & Elite $M \pm \mathrm{SD}$ & Medium level $M \pm \mathrm{SD}$ & $P$ \\
\hline Weight $(\mathrm{kg})$ & $76.6 \pm 9.3$ & $82.3 \pm 10.4$ & \\
\hline Height $(\mathrm{m})$ & $1.81 \pm 0.05$ & $1.79 \pm 0.05$ & \\
\hline Age (years) & $22.3 \pm 4.5$ & $35.2 \pm 10.4$ & $<0.001$ \\
\hline Experience (years of practice) & $11.9 \pm 3.5$ & $14.6 \pm 2.8$ & $<0.05$ \\
\hline Correction of distance to the plastron & $0.06 \pm 0.29$ & $-0.06 \pm 0.08$ & \\
\hline
\end{tabular}


of the fencers to record the horizontal component of the reaction force $\left(F_{A X}\right.$ and $\left.F_{B X}\right)$. A system to film 3D movement (VICON-460 with six infrared video cameras), at $500 \mathrm{~Hz}$, recorded the spatial positions of the eight body markers situated on the two heals, the front tips of the feet, the knee (epicondylus fibularis femoris), the hip (trochanter major), the shoulder tuberculum majus, the elbow (epicondylus radialis) of the sword arm and three markers situated on the blade of the sword (first third, second third and point). To record the RRT, a digital $1 / 1000$ second stopwatch was adapted to a system wired to the sword that recorded each time the sword point touched the plastron (surface on which the sword hits, allowing a certain degree of absorption of the impact, hereafter touché). A projector connected to a computer with an external programmable card timed the projection of a black circle $(0.09 \mathrm{~m})$ onto a white surface $(0.70 \times 0.55 \mathrm{~m})$ that served as the plastron. For the synchronisation of all the systems, an electronic signal enabled a simultaneous start.

\section{Procedure}

After a general warm-up and precise instructions for the task to be performed, the fencers were positioned en garde with one foot on each of the platforms. Afterwards, the projection screen or plastron was moved according to the size of the fencer, where the centre of the plastron was situated at shoulder height of the fencer and the distance between the front tip of the rear foot and the plastron was 1.5 -fold the height of the fencer. After several lunges against the plastron at the pre-established distance, the fencers were allowed to make several spatial adjustments needed to feel comfortable in terms of distance. Next the distance to the projector was adjusted with respect to the plastron so that the circle projected had the pre-established dimension $(0.09 \mathrm{~m}$ in diameter). After the adjustments, the elite group increased the distance of the plastron a mean of $0.06 \pm 0.29 \mathrm{~m}$, the medium-level group approximately $-0.06 \pm 0.08 \mathrm{~m}$ (see Table $\mathrm{I}$, in which the negative sign indicates proximity to the plastron).

After the announcement of ready, and a random preparatory time period of between 0.5 and $1.2 \mathrm{~s}$, a button was pressed to project the circle onto the centre of the plastron and start recording in all the systems $\left(E_{0}\right)$. At this point, the fencer had to lunge as swiftly as possible, attempting to situate the point of the sword inside the circle (touché). After several repeats for the fencers to become accustomed to the situation, five trials were carried out, valid for all the fencers and the errors were noted when the point of the sword did not reach the circle. The RT was defined as the period between the appearance of the circle in the centre of the plastron $\left(\mathrm{E}_{0}\right)$ until the start of the movement $\left(t_{0}\right)$.
Following the methodology proposed by GutiérrezDávila, Dapena, and Campos (2006), $t_{0}$ was determined from the instant in which the net force of the horizontal component $\left(\mathrm{F}_{\mathrm{AX}}+\mathrm{F}_{\mathrm{BX}}\right)$ reached a value higher than or equal to $1 \%$ of the fencer's body weight. If this time was less than $100 \mathrm{~ms}$, the trial was repeated. The movement time (MT) was measured from the RRT, recorded by the timer connected to the switch on the sword (MT $=$ RRT $-\mathrm{RT}$ ).

After the recording of the five valid trials corresponding to the above situation, the trials with target change were performed. As in the previous situation, beginning from the en garde situation, the fencer lunged as rapidly as possible to situate the point of the sword within the circle that appeared in the centre of the plastron. Before the end of the reaction response, the position of the circle could be randomly changed, or not, towards three equidistant positions, which were considered to be a second stimulus $\left(\mathrm{E}_{\mathrm{C}}\right)$. The three positions were situated on both sides of the lower zone, at $0.20 \mathrm{~m}$ of the starting position (circle situated in the centre of the plastron). The instant at which there was a change in stimulus position, denoted as 'stimulus change' $\left(t_{\mathrm{SC}}\right)$, was prefixed for each subject. The $t_{\mathrm{SC}}$ was calculated using the median RRT of the five valid trials of the preceding phase, where $t_{\mathrm{SC}}=\mathrm{RT}+(\mathrm{MT} / 4)$.

To avoid the learning factor, nine trials were made under different conditions: five valid trials in which the stimulus was changed at that very moment $t_{\mathrm{SC}}$, two without changing the stimulus and two changing the stimulus at a random time between RT and $t_{\mathrm{SC}}$. The order of the trials was random, repeating the trials that did not reach the target, and recording the error. Only the five valid trials where the target was changed in $t_{\mathrm{SC}}$ were registered, and only the median record was analysed with respect to RRT.

\section{Data analysis}

For the records of the RT and MT, the same procedure was used as in the previous phase. Due to the target changes during the MT, this was divided into three time periods. The first was called 'MTstimulus change', defined as the period between the start of the movement $\left(t_{0}\right)$ and the instant of the stimulus change $\left(t_{\mathrm{SC}}\right)$. The second was the CRT, defined as the time period between the $\mathrm{t}_{\mathrm{SC}}$ and the instant when the fencer changes the direction of the sword towards the new target $\left(t_{\mathrm{TC}}\right)$. The $t_{\mathrm{TC}}$ was determined using the change in the transverse or vertical component of the acceleration of the marker situated at the second third of the sword (depending on whether the movement of the target was lateral or downwards, respectively). Although the marker situated on the point of the sword was more sensitive to change, this was rejected on considering that the 
movements due to the elasticity of the foil could cause certain interferences. For the calculation of the acceleration components, the second derivate of the movement vector was used through fifth-grade splines without applying the smoothing function. Finally, the third period was the 'MT-target change', defined as the time period between the $t_{\mathrm{TC}}$ and the instant of a touché. Figure 2 provides a temporal scheme of the RRT for the situation with a target change.

For the sequence of the segmented participation to be determined, times were recorded when the maximum horizontal velocity was reached by the markers situated at the beginning of the forte of the blade and on the heel of the more advanced foot. The time of maximum arm extension (time $V_{\mathrm{x}(\mathrm{MAX})}$ arm extension) was recorded after the first peak of horizontal velocity registered in the marker situated at the beginning of the forte. The times of the maximum foot and sword velocity (Time $V_{\mathrm{X} \text { (MAX) }}$ foot and Time $V_{\mathrm{X}(\mathrm{MAX})}$ sword, respectively) correspond to the maximum horizontal velocity reached by the marker situated on the heel of the more advanced foot and on the one situated at the beginning of the forte, respectively. For each moment, a record of horizontal velocities regarding the time for the indicated markers, as well as for the centre of mass $(\mathrm{CM})$, is presented in Figure 3.

From the data recorded from the force platforms, records related to velocities and movements of the $\mathrm{CM}$ were determined, following the methodology proposed by Gutiérrez-Dávila, Dapena, and Campos (2006). The horizontal acceleration of the CM (Center of Mass of the fencer) was calculated from the net horizontal force of the two platforms $\left(\mathrm{F}_{\mathrm{AX}}\right.$ and $\left.\mathrm{F}_{\mathbf{B X}}\right)$ and the mass of the fencer. Progressive horizontal velocities $\left(\boldsymbol{v}_{\mathrm{CM}(\mathrm{X})}\right)$ and displacements $\left(\boldsymbol{s}_{\mathrm{CM}(\mathrm{X})}\right)$ were calculated from the horizontal acceleration-time values using trapezoidal integration. For the calculation of the components of velocity and horizontal shifts of the markers, the first derivate was applied to the respective components of position with respect to time, through fifth-grade splines without applying the smoothing function. The angles of the flexion of the elbow $\left(\boldsymbol{\theta}_{\text {(ELBOW })}\right)$ and the shoulder ( $\boldsymbol{\theta}_{\text {(SHOULDER) }}$ ) were calculated using the scalar product of the vectors that determined the positions of the markers defining the respective segments, (shoulder-hip/shoulder-elbow, for the shoulder and elbow-shoulder/elbow-shoulder/elbow angle-beginning of the forte of the blade, for the elbow angle). The length of the lunge was defined as the distance from the marker of the front tip of the rear foot in the en garde position to the marker situated at the heal of the front foot when the foot was firmly planted on the floor after the lunge.

Data were assessed for normality and homogeneity of variance, and are expressed as mean and standard deviation (SD) for each experimental variable and group. We used Software Statgraphics 5.1 of Statistical Graphics Corporation (STCS, Inc., Rockville, $M D$, USA) to treat the data. A one way ANOVA was used to quantify the differences between the average scores in the two experimental groups. The level for acceptance of significance $(\alpha)$ was set at 0.05 .

\section{Results}

Table II presents the temporal parameters of the RRT for the elite and medium-level group. The results show that the mean time of the flight phase was significantly greater for the elite group $(36 \pm 37 \mathrm{~ms}$ vs. $-2 \pm 12 \mathrm{~ms}$ ). The negative values registered for the medium-level group indicate that the airborne phase did not exist and that the front foot made contact with the floor a mean of $2 \mathrm{~ms}$ before ending the acceleration phase. For the rest of the temporal variables, significant statistical differences were not detected.

The errors are expressed in percentages of all the trials made in the situation of changing targets. Thus, the medium-level group committed a higher percentage of errors than did the elite group $(43 \pm 12 \%$ vs. $31 \pm 17 \%)$. The horizontal velocity $\left(v_{\mathrm{CMx}}\right)$ and movement of the $\mathrm{CM}\left(\boldsymbol{s}_{\mathrm{CMx}}\right)$ at the end of the acceleration phase were significantly greater for the elite group $\left(1.93 \pm 0.33 \mathrm{~m} \cdot \mathrm{s}^{-1}\right.$ vs. $1.39 \pm$ $0.22 \mathrm{~m} \cdot \mathrm{s}^{-1}$ and $0.41 \pm 0.07 \mathrm{~m}$ vs. $0.32 \pm 0.09 \mathrm{~m}$,

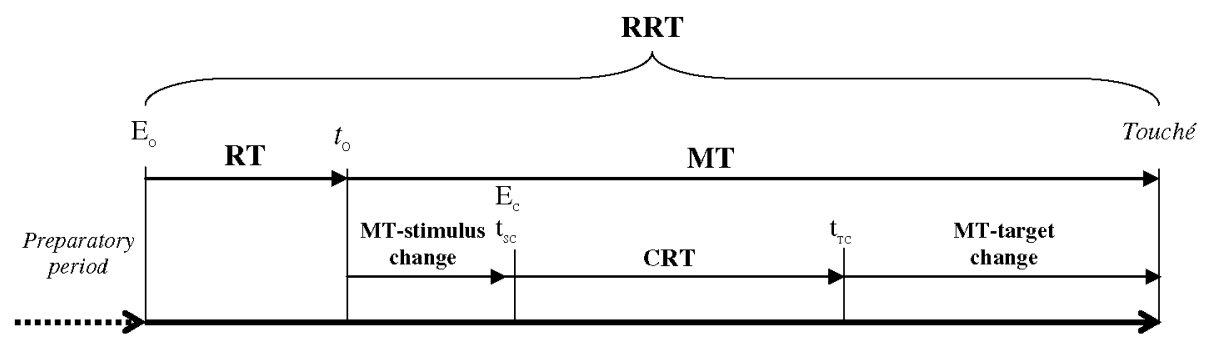

Figure 2. Scheme of the timing variables analysed in the response reaction for the situation surrounding a target change: choice reaction time (CRT), initial stimulus $\left(\mathrm{E}_{\mathrm{o}}\right)$, second stimulus $\left(\mathrm{E}_{\mathrm{C}}\right)$, movement time (MT), Movement time-stimulus change (MT-stimulus change), Movement time-target change (MT-target change), response reaction time (RRT), reaction time (RT), instant of the stimulus change $\left(t_{\mathrm{SC}}\right)$, instant when the fencer changes the direction of the sword towards the new target $\left(t_{\mathrm{TC}}\right)$. 


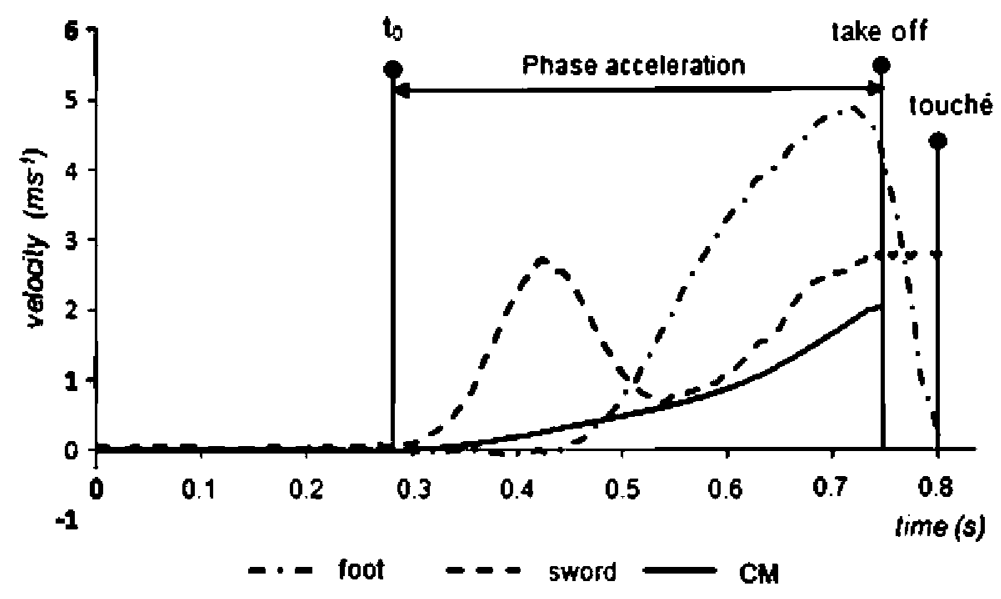

Figure 3. Velocity-time graphic when making a lunge attack and change of target for foot markers (heel) and weapon (marker situated at the beginning of the forte of the blade) and centre of mass (CM).

respectively), while no significant differences were found for $v_{\mathrm{CMx}}$ or $\boldsymbol{s}_{\mathrm{CMx}}$ for the instants of the stimulus change $\left(t_{\mathrm{SC}}\right)$ or the target change $\left(t_{\mathrm{TC}}\right)$. Consequently, the differences recorded in $\boldsymbol{v}_{\mathrm{CMx}}$ and $\boldsymbol{s}_{\mathrm{CMx}}$, at the end of the acceleration phase occurred as a result of what occurred in the MT-target change.

Table III presents certain variables that describe the starting position, en garde, for the two groups. The time records were expressed with respect to the percentage of the duration of the acceleration phase. For none of the variables that describe the en garde positions were significant statistical differences found between the mean of the two groups. Consequently, the mean starting position proved similar for the two groups.

During the initial thrust of the arm, the elite group achieved maximum horizontal velocity of the sword $\left(v_{\mathrm{X}(\operatorname{MAX})}\right.$ sword; marker situated at the beginning of the first third) sooner than did the mediumlevel group ( $31 \pm 13 \%$ vs. $45 \pm 20 \%$ ). Nevertheless, no statistically significant differences were found in the maximum horizontal velocity reached by the sword ( $v_{\mathrm{X}(\mathrm{MAX})}$ arm extension), either in the extension degree of the elbow or shoulder angle at the same moment $\boldsymbol{\theta}_{\text {(ELBOW) }}$ maximum extension velocity and $\boldsymbol{\theta}_{\text {(SHOULDER) }}$ maximum extension velocity, respectively).

The elite group reached the highest horizontal foot velocity ( $v_{\mathrm{X}(\mathrm{MAX})}$ foot) (marker situated on the heel of the more advanced foot) later than did the medium-level group $(75 \pm 5 \%$ vs. $58 \pm 10 \%)$. At that moment ( $v_{\mathrm{X}(\mathrm{MAX})}$ foot), the average sword horizontal velocity was higher for the elite group $\left(4.56 \pm 0.75 \mathrm{~m} \cdot \mathrm{s}^{-1}\right.$ vs. $\left.3.59 \pm 0.30 \mathrm{~m} \cdot \mathrm{s}^{-1}\right)$, the elbow reached greater extension $\left(159 \pm 11^{\circ}\right.$ vs. $141 \pm 18^{\circ}$ ); and the shoulder maintained greater flexion $\left(99 \pm 13^{\circ}\right.$ vs. $\left.78 \pm 16^{\circ}\right)$. Consequently, the elite group achieved an arm-foot timing sequence while the medium-level group moved the sword and front foot simultaneously forward.

No differences in timing were found regarding the horizontal velocity of the sword (Time $v_{\mathrm{X}(\mathrm{MAX})}$ sword), although the horizontal velocity of the sword at this instant $\left(v_{\mathbf{X}}\right.$ (MAX) sword) was

Table II. Descriptive and inferential statistics of the timing parameters of the reaction response and certain technical factors for the group of elite and medium-level fencers

\begin{tabular}{lcc}
\hline Variables & Elite $M \pm \mathrm{SD}$ & Medium level $M \pm \mathrm{SD}$ \\
\hline Reaction time, RT (ms) & $220 \pm 32$ & $214 \pm 26$ \\
Movement time, MT (ms) & $601 \pm 82$ & $585 \pm 72$ \\
Response reaction time, RRT (ms) & $822 \pm 94$ & $798 \pm 73$ \\
Movement time-stimulus change, MT- $t_{\mathrm{SC}}(\mathrm{ms})$ & $167 \pm 47$ & $154 \pm 47$ \\
Choice reaction Time, CRT (ms) & $238 \pm 45$ & $232 \pm 30$ \\
Movement time target change, MT- $t_{\mathrm{TC}}(\mathrm{ms})$ & $212 \pm 58$ & $202 \pm 37$ \\
Time acceleration phase (ms) & $558 \pm 85$ & $510 \pm 76$ \\
Time flight phase (ms) & $36 \pm 37$ & $-2 \pm 12$ \\
Errors $(\%)$ & $31 \pm 17$ & $43 \pm 12$ \\
Displacement of CM, $S_{\mathrm{CMx}}$ at $t_{\mathrm{SC}}(\mathrm{m})$ & $0.02 \pm 0.02$ & $0.02 \pm 0.01$ \\
Horizontal velocity of CM, $V_{\mathrm{CMx}}$ at $t_{\mathrm{SC}}\left(\mathrm{m} \cdot \mathrm{s}^{-1}\right)$ & $0.25 \pm 0.21$ & $0.25 \pm 0.14$ \\
Displacement of $\mathrm{CM}, S_{\mathrm{CMx}}$ at $t_{\mathrm{TC}}(\mathrm{m})$ & $0.16 \pm 0.10$ & $0.17 \pm 0.06$ \\
Horizontal velocity of $\mathrm{CM}, V_{\mathrm{CMx}}$ at $t_{\mathrm{TC}}\left(\mathrm{m} \cdot \mathrm{s}^{-1}\right)$ & $1.14 \pm 0.58$ & $1.06 \pm 0.25$ \\
Displacement of $\mathrm{CM}, S_{\mathrm{CMx}}$ in the acceleration phase $(\mathrm{m})$ & $0.41 \pm 0.07$ & $0.32 \pm 0.09$ \\
Horizontal velocity of $\mathrm{CM}, V_{\mathrm{CMx}}$ at end of the acceleration phase $\left(\mathrm{m} \cdot \mathrm{s}^{-1}\right)$ & $1.93 \pm 0.33$ & $1.39 \pm 0.22$ \\
\hline
\end{tabular}


Table III. Descriptive and inferential statistics of certain kinematic variables for the elite and medium-level groups of fencers

\begin{tabular}{|c|c|c|c|}
\hline Variables & Elite $M \pm \mathrm{SD}$ & Medium level $M \pm \mathrm{SD}$ & $P$ \\
\hline \multicolumn{4}{|l|}{ En garde position } \\
\hline Horizontal hip position (m) & $0.37 \pm 0.05$ & $0.35 \pm 0.08$ & \\
\hline Horizontal shoulder position $(\mathrm{m})$ & $0.52 \pm 0.09$ & $0.47 \pm 0.09$ & \\
\hline Initial horizontal foil position (m) & $1.07 \pm 0.10$ & $1.03 \pm 0.13$ & \\
\hline Elbow angle, $\left.\boldsymbol{\theta}_{\text {(ELBOw) }}{ }^{\circ}\right)$ & $109 \pm 14$ & $110 \pm 14$ & \\
\hline Shoulder angle, $\left.\boldsymbol{\theta}_{\text {(SHOULDER) }}{ }^{\circ}\right)$ & $45 \pm 13$ & $47 \pm 18$ & \\
\hline \multicolumn{4}{|l|}{ Movement time } \\
\hline Time $v_{x(\operatorname{MAX})}$ arm extension (\%) & $31 \pm 13$ & $45 \pm 20$ & $<0.05$ \\
\hline$v_{\mathrm{X}} \operatorname{arm}$ extension $\left(\mathrm{m} \cdot \mathrm{s}^{-1}\right)$ & $1.82 \pm 0.3$ & $1.82 \pm 0.46$ & \\
\hline $\boldsymbol{\theta}_{(\mathrm{ELBOW})}$ at maximum velocity of the arm extension $\left(^{\circ}\right)$ & $123 \pm 10$ & $124 \pm 13$ & \\
\hline $\boldsymbol{\theta}_{(\mathrm{SHOULDER})}$ at maximum velocity of the $\operatorname{arm}$ extension $\left({ }^{\circ}\right)$ & $65 \pm 9$ & $64 \pm 13$ & \\
\hline Time $V_{\mathbf{X}(\operatorname{MAX})}$ foot $(\%)$ & $75 \pm 5$ & $58 \pm 10$ & $<0.001$ \\
\hline$v_{\mathrm{X}(\mathrm{MAX})}$ foot $\left(\mathrm{m} \cdot \mathrm{s}^{-1}\right)$ & $4.56 \pm 0.75$ & $3.59 \pm 0.30$ & $<0.001$ \\
\hline$\theta_{\text {(ELBOw) }}$ at maximum velocity of the foot $\left(^{\circ}\right)$ & $159 \pm 11$ & $141 \pm 18$ & $<0.05$ \\
\hline$\theta_{\text {(SHOULDER })}$ at maximum velocity of the foot $\left({ }^{\circ}\right)$ & $99 \pm 13$ & $78 \pm 16$ & $<0.005$ \\
\hline Time $V_{X(\text { MAX })}$ sword $(\%)$ & $87 \pm 8$ & $87.2 \pm 9$ & \\
\hline$v_{\mathrm{X}(\mathrm{MAX})}$ sword $\left(\mathrm{m} \cdot \mathrm{s}^{-1}\right)$ & $2.55 \pm 0.42$ & $1.88 \pm 0.48$ & $<0.001$ \\
\hline $\boldsymbol{\theta}_{\text {(ELBOw) }}$ at maximum velocity, sword $\left(^{\circ}\right)$ & $160 \pm 11$ & $153 \pm 12$ & \\
\hline $\boldsymbol{\theta}_{\text {(SHOULDER })}$ at maximum velocity, sword $\left({ }^{\circ}\right)$ & $105 \pm 11$ & $90 \pm 9$ & $<0.001$ \\
\hline$v_{\mathrm{X}}$ sword at touché $\left(\mathrm{m} \cdot \mathrm{s}^{-1}\right)$ & $2.28 \pm 0.47$ & $1.74 \pm 0.54$ & $<0.01$ \\
\hline Length of lunge $(\mathrm{m})$ & $1.40 \pm 0.15$ & $1.13 \pm 0.13$ & $<0.001$ \\
\hline
\end{tabular}

Notes: Time $V_{\mathrm{X}(\mathrm{MAX})}$ arm extension (\%): beginning or arm extension.

$v_{\mathrm{X}}$ arm extension $\left(\mathrm{m} \cdot \mathrm{s}^{-1}\right)$ : sword horizontal velocity at beginning of arm extension.

Time $V_{\mathrm{X}(\mathrm{MAX})}$ foot $(\%)$ : necessary time for the advanced foot to reach the maximum velocity.

$V_{\mathrm{x}(\operatorname{MAX})}$ foot $\left(\mathrm{m} \cdot \mathrm{s}^{-1}\right)$ : foot maximum horizontal velocity.

Time $V_{\mathrm{x}(\text { MAX })}$ sword (\%) necessary time to reach maximum horizontal velocity in the sword

$v_{\mathrm{X}(\operatorname{MAX})}$ sword $\left(\mathrm{m} \cdot \mathrm{s}^{-1}\right)$ : sword maximum velocity.

$v_{\mathrm{X}}$ sword at touché $\left(\mathrm{m} \cdot \mathrm{s}^{-1}\right)$ : sword velocity at touché.

significantly greater for the elite group. This trend was maintained until the instant of the touché, although with somewhat lower mean records $\left(2.55 \pm 0.42 \mathrm{~m} \cdot \mathrm{s}^{-1}\right.$ vs. $\left.1.88 \pm 0.48 \mathrm{~m} \cdot \mathrm{s}^{-1}\right)$. The two groups maintained an extended elbow similar at the instant of reaching the maximum velocity of the sword, while the angle of the shoulder was significantly higher for the elite group $\left(105 \pm 11^{\circ}\right.$ vs. $\left.90 \pm 9^{\circ}\right)$. Finally, the elite group achieved a greater mean length of the lunge compared to the mediumlevel group $(1.40 \pm 0.15 \mathrm{~m}$ vs. $1.13 \pm 0.13 \mathrm{~m})$.

\section{Discussion}

The fact that the age of the elite group was significantly lower than that of the medium level group $(P<0.001$ - see Table I) can be explained due to the participation of the elite group of six fencers who belonged to the National Junior team (younger than 20 years old). When experience was quantified by the years of practice, the differences registered between the groups in terms of the experience $(P<0.05)$ was also conditioned by the young age of the six junior fencers included in the elite group. Considering that the average age for the 24 first fencers classified in the World Ranking of men's sword competition in 2011 was $30 \pm 4$ years, it is not surprising to see the older age registered in the medium-level group $(35.2 \pm 10.4$ years $)$.
The results indicate that the elite and mediumlevel sword fencers did not differ with respect to simple RT, and reaction time of target change (CRT). The data contrast with the findings of Williams and Walmsley (2000a, b) and of Di Russo et al. (2006), who reported that elite fencers were faster in RT and MT than were novices or students who had not practiced fencing. Thus, the differences regarding the results in this work could be due to the large differences in the amount of practice of the groups (Schmidt \& Lee, 1999; Sosnoff \& Samson, 2007; Utley \& Astill, 2008).

On the other hand, on the basis of the two-visualsystem model proposed by Milner and Goodale (2008), in which two independent visual systems interact to gather information for different purposes, the RT and CTR, the ventral stream would primarily gather information close to action (affordance), possibly more related to the performance of beginner sportsmen, while the precision and execution of the movement would be dominated by a dorsal stream, this offering information related to movement control, which would be typical of sportsmen with a high degree of automatism (van der Kamp, Rivas, van Doorn, \& Savelsbergh, 2008). Our data support this theory, reflecting that the elite group is more precise in its technical execution than was the medium-level group (see Table II). This will be discussed below. As opposed to previous research, the complexity of the 
task proposed in the present study resides in the fact that the fencer had to restrain the initial motor activity and change the target while accelerating forward. According to the data presented in Table II, the fencers required a mean of $40 \%$ of the MT to process the information (CRT). However, no significant statistical differences were found between groups for MT. The only differences were found in the time needed for the flight phase. The mediumlevel group put their foot on the floor an average of 2 $\mathrm{ms}$ before they finished the acceleration phase. Considering that the time of movement averaged $76 \pm 54 \mathrm{~ms}$ higher for the acceleration phase (MTTime acceleration phase). Thus, the medium-level group made the touche when the $\mathrm{CM}$ was already reducing their velocity (braking phase), while the elite group did so at the end of the flight phase. The fact that the medium-level group did not have a phase in the air and the mean length of the lunge was shorter than in the elite group suggests that there was a precipitated support of the front foot when lunging.

Although the position and velocity of $\mathrm{CM}$ at target contact were underestimated for the medium-level subjects, the consequences of premature planting of the foot on the floor were: (1) the acceleration phase was interrupted, allowing less velocity and horizontal movement of the $C M$ in the final phase of acceleration, a fact confirmed by the significantly higher values reached by the elite group for these two variables (see Table I); (2) the horizontal velocity of the CM during the time period necessary to change target was reduced, this being confirmed by the differences found in the horizontal velocity of the $\mathrm{CM}$ at the end of the acceleration phase during the MT-target change; and (3) the instant of the touche occurred when the $\mathrm{CM}$ was already reducing the horizontal velocity (during the braking phase). As a consequence, the horizontal velocity of the sword at that instant had to be reduced. This fact is confirmed both by the maximum horizontal velocity of the sword ( $\mathbf{v}_{\mathrm{X} \text { (MAX) }}$ sword), as well as the reach at the instant of the touché ( $v_{\mathrm{X}}$ sword at touché), being significantly longer for the elite group (Table III).

The differences between the groups, with respect to the variables used to describe the sequence of the segmented participation (Table III), show that the medium-level group made a simultaneous forward movement of the foot and sword arm whereas the elite group executed a temporal arm-foot sequence. As a result, the elite were quicker to reach maximum velocity in the initial extension of the arm (31\% vs. $45 \%$ of the MT) and achieve maximum horizontal velocity of the foot later ( $75 \%$ vs. $58 \%$ of the $\mathrm{MT}$ ). These results confirm the findings of Klinger and
Adrian (1983), as well as, Stewart and Kopetka (2005), highlighting the importance of starting the advance with a rapid thrust of the arm followed by a lunge forward with the front foot. The temporal arm-foot sequence is required for a correct technique and also determines the right of way (priorite) in foil and sabre competitions.

Another technical aspect to be taken into account is that the elite group, apart from achieving the maximum velocity of the front foot later, also registered a significantly higher value of foot velocity than that reached by the medium-level group. This suggests a greater contribution of the rear leg to horizontal acceleration of the $\mathrm{CM}$, at least up to $75 \%$ of the MT and during the MT-target change. On the contrary, the medium-level group attained maximum velocity of the foot at $58 \%$ of the MT, while the target change takes $386 \pm 52 \mathrm{~ms}$, after starting the movement $(66 \pm 6 \%$ of the MT). Therefore, the forward action of the leg would end its contribution to the horizontal acceleration of the CM before starting the target change, justifying its lower increase in horizontal velocity of the CM during the MT-target change.

The changes detected in the technical execution of the two groups coincide with the findings of Yiou and Do (2000), who report that elite fencers achieve superior performance and velocity of $\mathrm{CM}$ in complex attack movements. Although different methodologies would be used, our results could strengthen the contributions of Williams and Walmsley (2000b) indicating that, in long- or medium-distance lunges, expert fencers attain more coherent muscle synergy and a more consistent pattern of muscle coordination than do beginning fencers.

Finally, the present work shows that the coordination of fencing movements, that is, the right technique, constitutes a factor that differentiates elite fencers from medium-level ones. Therefore, we emphasise the importance of training the right technical execution of the fencing movements also in complex situations. However, we question the effectiveness of training oriented exclusively towards improving the timing of information processing. This aspect appears to improve with practice, but has not been confirmed to differentiate elite from medium-level fencers.

\section{Acknowledgements}

The authors wish to thank Cristina López de Subijana for her invaluable help collecting data, and the Spanish Royal Federation of Fencing for its collaboration in this project, providing access to the fencers of the National Sword fighting Team. 


\section{References}

Borysiuk, Z., \& Cynarski, W. J. (2010). Psychomotor aspects of talent identification: A new approach in the case of fencing. Archives of Budo, 6(2), 91-94.

Borysiuk, Z., \& Waskiewicz, Z. (2008). Information processes, stimulation and perceptual training in fencing. Fournal of Human Kinetics, 19, 63-82.

Di Russo, F., Taddei, F., Aprile, T., \& Spinelli, D. (2006). Neural correlates of fast stimulus discrimination and response selection in top-level fencers. Neuroscience Letters, 408, 113-118.

Dogan, B. (2009). Multiple-choice reaction and visual perception in female and male elite athletes. fournal of Sports Medicine and Physical Fitness, 49(1), 91-96.

Guizani, M. S., Tenenbaum, G., Bouzaouach, I., Ben-Kheder, A., Feki, Y., \& Bouaziz, M. (2006). Information-processing under incremental levels of physical loads: Comparing racquet to combat sports. Fournal of Sports Medicine and Physical Fitness, $46,335-435$.

Gutiérrez-Dávila, M., Dapena, J., \& Campos, J. (2006). The effect of muscular pre-tensing on the sprint start. fournal of Applied Biomechanics, 22(3), 194-201.

Klinger, A., \& Adrian, M. (1983). Foil target impact forces during the fencing lunge. In H. Matsui, \& K. Kobayashi (Eds.), Biomechanics, VIII-B: Proceedings of the Eighth International Congress of Biomechanics Series of Biomechanics (pp. 882-888). Nagoya, Japan and Champaign, IL: Human Kinetics.
Milner, A. D., \& Goodale, M. A. (2008). Two visual systems reviewed. Neuropsychologia, 46, 774-785.

Schmidt, R. A., \& Lee, T. D. (1999). Motor control and learning (3rd ed, p. 306. Champaign, IL: Human Kinetics.

Sosnoff, J. J., \& Samson, J. (2007). Physical activity and variability of cognitive processing. Fournal of Sport and Exercise Psychology, 29 (Suppl.), S22-S23.

Stewart, S. L., \& Kopetka, B. (2005). The kinematic determinants of speed in the fencing lunge. Fournal of Sports Sciences, $23(2), 105$.

Utley, A., \& Astil, S. (2008). Motor control, learning and development (pp. 64-72). London: Taylor and Francis.

van der Kamp, J., Rivas, F., van Doorn, H., \& Savelsbergh, G. (2008). Ventral and dorsal contributions in visual anticipation in fast ball sports. International fournal of Sport Psychology, 39, $100-130$.

Williams, L. R. T., \& Walmsley, A. (2000a). Response amendment in fencing: Differences between elite and novice subjects. Perceptual and Motor Skills, 91(1), 131-142.

Williams, L. R. T., \& Walmsley, A. (2000b). Response timing and coordination in fencing: A comparison of elite and novice fencers. Fournal of Science and Medicine in Sports, 3(4), 460475.

Yiou, E., \& Do, M. C. (2000). In fencing, does intensive practice equally improve the speed performance of the touché when it is performed alone and in combination with the lunge. International fournal of Sport Medicine, 21(2), 122-126. 